Bull. Egypt. Soc. Physiol. Sci. Vol. (42), Issue (1), 11-26

\author{
Bull. of Egyp. Soc. Physiol. Sci. \\ (Official Journal of Egyptian Society for Physiological Sciences) \\ (pISSN: 1110-0842; eISSN: 2356-9514)
}

\title{
Neuroprotective Effect of Piracetam and Vincamine in a Rat Model of Haloperidol- induced Parkinson's Disease
}

\author{
Alzahraa A Sheref ${ }^{1 *}$, Yahya M Naguib ${ }^{1,2}$, Elsayed S Abou-Elnour ${ }^{3}$, Heba R Salem ${ }^{1}$, Mohammed HA \\ Hassan $^{1}$, Hesham AD Abdel-Razek ${ }^{1}$
}

${ }^{1}$ Medical Physiology Department, Faculty of Medicine, Menoufia University, Shebein El-Kom, Menoufia Governorate, Egypt.

${ }^{2}$ Physiology Department, College of Medicine and Medical Sciences, Arabian Gulf University, Manama, Bahrain.

${ }^{3}$ Biochemistry Departments, Faculty of Medicine, Menoufia University, Shebein El-Kom, Menoufia Governorate, Egypt.

Submit Date: 5 April 2021

Revise Date: 12 June 2021

Accept Date : 30 July 2021

\section{Keywords}

Parkinson's

Disease

Haloperidol

Piracetam

Vincamine

\begin{abstract}
To evaluate the neuroprotective effect of nootropic drugs, piracetam and vincamine, on Parkinson's disease (PD) in rats, forty adult male Wistar albino rats were randomized into five equal groups: control, haloperidol-induced PD group, and PD groups orally given piracetam (300 $\mathrm{mg} / \mathrm{kg} /$ day $)$, vincamine $(20 \mathrm{mg} / \mathrm{kg} /$ day $)$ or both. Four weeks later, motor performance was assessed by stepping test. Y-maze, forced swimming and olfactory preference tests were done for cognitive and behavioral evaluation. Blood samples were collected for measuring serum glucose, calcium, creatine phosphokinase (CPK) and glial cell-derived neurotrophic factor (GDNF). Thereafter, rats were sacrificed and brains were dissected. Striatal tissue of left hemisphere was isolated and homogenized for assay of dopamine, malondialdehyde (MDA), nitrite/nitrate, reduced glutathione (GSH), glutathione peroxidase (GPx), superoxide dismutase (SOD), tumor necrosis factor-alpha (TNF- $\alpha$ ) and interleukin-1beta (IL-1ß). Right hemisphere was used for histopathological examination of substantia nigra. Results: Rats of PD group showed bradykinesia, cognitive impairment, depressive-like behavior and hyposmia, reductions in serum calcium and GDNF, and in striatal dopamine, GSH, GPx and SOD, while serum glucose and $\mathrm{CPK}$, and striatal MDA, nitrite/nitrate, IL- $1 \beta$ and TNF- $\alpha$ were increased, as compared to control. Both drugs improved neurological dysfunction and biochemical parameters, as compared to PD group. The histopathological findings revealed neuro-degeneration and neuro-inflammation in PD group, that improved in treated groups. The piracetam effect was mainly anti-inflammatory, while vincamine was mainly antioxidant. Combined treatment resulted in a more potent amelioration of haloperidol-induced changes. Conclusion: Piracetam and vincamine exhibit neuroprotective activity in haloperidol-induced PD, that is more potent with their combination.
\end{abstract}

Corresponding author: Alzahraa A Sheref, Medical Physiology Department, Faculty of Medicine, Menoufia University, Menoufia, Egypt. Tel:+20482222731, Fax: +20482317508, E-mail: Dr.zahraaelsherefma@gmail.com 


\section{INTRODUCTION}

Parkinson's disease (PD) is a common neurodegenerative disorder affecting over ten million people worldwide [1]. It is accompanied by progressive loss of dopaminergic neurons in the substantia nigra pars compacta, resulting in significant impairment of dopamine release [2]. Patients with PD are presented by motor and nonmotor symptoms. The traditional motor symptoms include bradykinesia, tremor, rigidity and postural instability [3]. Non-motor symptoms of PD include olfactory dysfunction, memory impairment, sleep disorders, anxiety and depression [4].

The underlying causes of PD include environmental factors [5], genetic factors [6] and drug-induced ones. Haloperidol is a classic antipsychotic drug, which causes Parkinson-like syndrome and extrapyramidal symptoms [7]. It can be used to induce experimental model of PD through blockage of the dopaminergic D2 receptors [8].

Nootropic drugs may have a role in protection and amelioration of the associated symptoms of PD, being a neurodegenerative disease. The metabolic enhancer piracetam, a cyclic derivative of gammaaminobutyric acid, was claimed to have neuroprotective effect in many neurological disorders, as it enhances membrane fluidity, cognitive function and neurotransmission [9]. Vincamine, a plant alkaloid, can be used clinically in the therapy of cerebral and circulatory disorders [10].

The aim of the present study was to investigate a neuroprotective effect of piracetam and vincamine on a haloperidol-induced rat model of PD, and whether or not their combined administration provides a better protection.

\section{Materials and Methods.}

\subsection{Experimental Animals and Design.}

All procedures were conducted in adherence to the Guide for the Care and Use of Laboratory Animals (8th edition, National Academies Press), with the approval of the Ethics Committee of Faculty of Medicine, Menoufia University, Egypt. Forty adult male Wister albino rats, weighing 160-200 grams each, were used in this study. Rats were kept on standard laboratory rat chow and water ad libitum. They were housed in cages measured 70x70x60 cm, 4 animals/cage under normal light/dark cycle at room temperature throughout the study period. Animals were left for one week for acclimatization, then they were randomly divided into five equal groups $(\mathrm{n}=8)$ : (i) Control $(\mathrm{C})$ group, in which rats were given $0.5 \mathrm{ml}$ of distilled water by oral gavage, and intraperitoneally (i.p) injected with $0.5 \mathrm{ml}$ of normal saline, once daily for 4 weeks. (ii) PD group, where rats were given $0.5 \mathrm{ml}$ of distilled water by oral gavage, once daily for 4 weeks, and i.p. injected with $5 \mathrm{mg} / \mathrm{kg}$ body weight of haloperidol (Nile Company for Pharmaceutical, Egypt) dissolved in $0.5 \mathrm{ml}$ of normal saline, once daily for 4 weeks [11]. (iii) Piracetam-treated PD (PD+Pir) group, where rats were given $300 \mathrm{mg} / \mathrm{kg}$ body weight of piracetam (Amoun Company, Egypt) [12] dissolved in $0.5 \mathrm{ml}$ of distilled water by oral gavage, once daily for 4 weeks, and they were i.p. injected with haloperidol as in PD group. (iv) Vincamine-treated PD (PD+Vin) group, where rats were given $20 \mathrm{mg} / \mathrm{kg}$ body weight of vincamine (Glaxo Company, Egypt) [13] dissolved in $0.5 \mathrm{ml}$ of distilled water by oral gavage, once daily for 4 weeks, and they were i.p. injected with haloperidol as in PD group. (v) Combined 
piracetam- and vincamine-treated PD (PD+Pir\&Vin) group, where rats received piracetam as in PD+Pir group and vincamine as in PD+Vin group, and they were injected with haloperidol as in PD group.

After 4 weeks-experimental period, rats underwent neurological and behavioral assessment tests, and then blood samples were collected. Then, rats were sacrificed by injection of lethal dose of thiopental sodium. Immediately after sacrification, rats were decapitated, and brains were carefully dissected. The basal ganglia of left hemispheres were isolated, and the striatal tissue was homogenized for biochemical analyses. The right hemispheres were preserved in $10 \%$ formalin to be used for histopathological studies.

\subsection{Neurological and behavioral assessment.}

Neurological and behavioral tests were done at room temperature in a calm room without any external interference.

\subsubsection{Stepping test.}

Stepping test was used to assess motor performance of rats. The rat was firmly suspended from its hindquarters, while it supported its weight on forelimbs. Then, the rat moved backward along the table $(0.9 \mathrm{~m}$ in 5 seconds $)$ three times repeatedly per session. For each session, the total calculated score was the sum of the number of adjusting steps observed in the three tests [14].

\subsubsection{Y-maze test.}

$\mathrm{Y}$-maze is considered a quick and relatively simple test of spatial working memory. The Y-maze used was made of wood with three arms of equal size $(60 \mathrm{~cm} \times 12 \mathrm{~cm} \times 25 \mathrm{~cm})$, converged at a $120^{\circ}$ angle with each other. Each rat was placed at the center of the maze and permitted to move freely for exploration of the arms for 8 min-duration. An arm entry was considered when the hind paws of the rat were completely within the arm. Any three successive entries of the three different arms were evaluated as a correct choice. Spontaneous alternation refers to the natural tendency of rodents to spontaneously choose alternate arms in a Ymaze. The percentage of spontaneous alternation was calculated as follows: (actual alternations/maximum alternations $) \times 100$. The number of maximum alternations equals total number of arms entered minus 2 [15].

\subsubsection{Forced swimming test.}

Rats were individually placed in cylinder $(15 \mathrm{~cm}$ in diameter and $40 \mathrm{~cm}$ in height), containing water. Immobility time and latency to immobility were evaluated during 5 min-period. Immobility was assigned when no additional activity observed other than that required to keep the rat head above the water. Depressive-like behavior was defined as an increase in the time of immobility and a decrease in latency to immobility [16].

\subsubsection{Olfactory preference test.}

The rats were presented small aliquots of either a novel scent (vanilla) or water simultaneously. Time spent for sniffing each odor is recorded for three minutes [17].

\subsection{Biochemical analysis.}

\subsubsection{Blood sampling and analysis.}

Fasting blood samples were collected from the retro-orbital venous plexus, using fine heparinized capillary tubes introduced into medial epicanthus of the rat's eye under light halothane inhalation anesthesia. The blood samples were left to clot for 15 minutes at room temperature. Then, they were centrifuged at 3000 r.p.m for 15 minutes, and the serum was separated and stored at $-20^{\circ} \mathrm{C}$ until used. Serum glucose and calcium levels was 
estimated using their specific kits (Biodiagnostic Company, Egypt). Serum creatine phosphokinase (CPK) level was measured using specific kit purchased from Biovision Incorporation (USA), according to manufacturer's instructions. Serum glial cell-derived neurotrophic factor (GDNF) level was measured using rat ELISA kit (My BioSource Incorporation, USA) and following the manufacturer's instructions.

\subsubsection{Striatal tissue homogenization and biochemical assay.}

Immediately after sacrification rats were decapitated. Brains were carefully removed and washed with ice-cold saline. The basal ganglia of the left hemisphere of each brain were isolated, and the striatal tissue was identified, weighed, and homogenized in 10\% (w/v) phosphate-buffered solution $(\mathrm{PH}=7)$, using glass rod. The homogenate was centrifuged at $2000 \times \mathrm{g}$ for $10 \mathrm{~min}$, and the supernatant was kept at $-80^{\circ} \mathrm{C}$ until biochemical analyses.

Striatal dopamine level was measured using a double-antibody sandwich ELISA kit (Sunred Company, Shanghai, China), according to the manufacturer's instructions. Striatal malondialdehyde (MDA) level was measured using its specific kit purchased from Biodiagnostic Company, Egypt. Nitrite/nitrate level in striatal tissue was estimated using colorimetric kit purchased from Biodiagnostic Company, Egypt, and following the manufacturer's instructions.

Striatal TNF- $\alpha$ and IL-1 $\beta$ levels were measured using the corresponding rat ELISA kits purchased from Sunred Company (Shanghai, China) and My BioSource Incorporation (USA), respectively, and following the manufacturer's instructions.
Striatal homogenate reduced glutathione (GSH) level was measured by its colorimetric kit purchased from Biodiagnostic Company (Dokki, Giza, Egypt). Striatal glutathione peroxidase (GPx), and superoxide dismutase (SOD) enzymatic activities were determined using their specific colorimetric kits (Biodiagnostic Company, Egypt), according to the manufacturer's instructions.

\subsection{Histopathological examination.}

Immediately after dissection, the right hemisphere of each rat brain was placed in $10 \%(\mathrm{v} / \mathrm{v})$ formalin for 24 hours. Tissues were washed in tap water, and serial dilutions of alcohol (methyl, ethyl, and absolute ethyl) were used for dehydration. Thereafter, specimens of substantia nigra pars compacta (SNpc) were cleared in xylene for 20-30 minutes and impregnated in soft paraffin wax for two hours, then in hard paraffin wax at room temperature. Finally, tissue blocks were formed and cut out into sections of five microns thickness, and then stained with hematoxylin and eosin stain (H\&E) [18]. After staining, the sections were examined and photographed using a bright-field microscope.

\subsection{Statistical analysis.}

All results were expressed as the mean \pm standard deviation (SD). The data were analyzed using SPSS program version 22.0 (SSPSS Inc., Chicago, IL, USA). One-way ANOVA, the F test, and post hoc Tukey's multiple comparison test were calculated to test the presence of significance between the studied groups. Correlation between variables was performed using Pearson coefficient. $\mathrm{P}$ value $\leq 0.05$ was considered statistically significant [19]. 


\section{Results.}

\subsection{Neurological and behavioral tests.}

A significant difference in the number of adjusting steps of stepping test was observed between the studied groups. The number of adjusting steps was significantly lower in $\mathrm{PD}$ group than in $\mathrm{C}$ group. The treated groups (PD+Pir, PD+Vin and PD+Pir\&Vin) showed significantly higher values, when compared with PD group, but were still significantly lower than that of $\mathrm{C}$ group. Combined piracetam and vincamine administration showed a significantly better improvement than their isolated administration (Fig 1-A).

The mean value of percentage of spontaneous alternation in Y-maze was significantly lower in PD group than in $\mathrm{C}$ group. The treated groups (PD+Pir, PD+Vin and PD+Pir\&Vin) showed significantly higher values, when compared with PD group, but were still significantly lower than that of $\mathrm{C}$ group. Combined treatment in PD+Pir\&Vin group showed significantly more percentage of spontaneous alteration than those of $\mathrm{PD}+\mathrm{Pir}$ and $\mathrm{PD}+\mathrm{Vin}$ groups; the latter groups showed nonsignificant variation from each other (Fig 1-B).

The duration of olfaction of the olfactory test was significantly lower in $\mathrm{PD}$ group than in $\mathrm{C}$ group. The treated groups (PD+Pir, PD+Vin and PD+Pir\&Vin) showed significantly higher values, when compared with PD group, but were still significantly lower than that of C group (Fig 1-C). PD+Pir\&Vin group showed a significantly more duration of olfaction than those of PD+Pir and $\mathrm{PD}+\mathrm{Vin}$ groups; however, $\mathrm{PD}+\mathrm{Pir}$ and $\mathrm{PD}+\mathrm{VIN}$ groups were insignificantly different.
Significant differences in latency and immobility durations of swimming test were found among the studied groups. The latency time was significantly lower in PD group than in $\mathrm{C}$ group. The treated groups (PD+Pir, PD+Vin and PD+Pir\&Vin) showed significantly higher values, when compared with PD group, but values were still significantly lower than that of $\mathrm{C}$ group. There was insignificant difference in latency time between PD+Pir and PD+Vin groups, but they were significantly lower than that of PD+Pir\&Vin group. The immobility time was significantly higher in PD group than in $\mathrm{C}$ group. The treated groups (PD+Pir, $\mathrm{PD}+\mathrm{Vin}$ and $\mathrm{PD}+\mathrm{Pir} \& \mathrm{Vin})$ showed significantly lower values, when compared with PD group, but values were still significantly higher than that of $\mathrm{C}$ group. $\mathrm{PD}+\mathrm{Pir}$ and $\mathrm{PD}+\mathrm{Vin}$ groups showed insignificant change in immobility time between each other. The immobility time of PD+Pir\&Vin group was significantly less than those of PD+Pir and PD+Vin groups (Fig 1-D).

\subsection{Biochemical analysis.}

\subsubsection{Serum biochemical measurements.}

Table (1) demonstrates the various biochemical parameters measured in the serum of all the studied groups.

Fasting serum glucose level was significantly higher in the $\mathrm{PD}$ group than in $\mathrm{C}$ group. In $\mathrm{PD}+\mathrm{Pir}, \mathrm{PD}+\mathrm{Vin}$ and $\mathrm{PD}+\mathrm{Pir} \& \mathrm{Vin}$ groups, serum glucose levels were significantly lower than that of PD group, but they were still significantly higher than that of $\mathrm{C}$ group. Nonsignificant variations in serum glucose levels were observed between the three treated groups.

Serum calcium level was significantly lower in PD group than in $\mathrm{C}$ group. In PD+Pir, PD+Vin and PD+Pir\&Vin groups, serum calcium levels were 
significantly elevated as compared to $\mathrm{PD}$ group. Serum calcium in PD+Pir\&Vin group was significantly higher than in PD+Vin group, but insignificantly changed as compared to PD+Pir group. The calcium levels in all treated groups were still significantly lower than that of $\mathrm{C}$ group.

Serum CPK level was significantly higher in PD group than in $\mathrm{C}$ group. In $\mathrm{PD}+\mathrm{Pir}, \mathrm{PD}+\mathrm{Vin}$ and PD+Pir\&Vin groups, CPK levels were significantly lower than that of PD group. PD+Pir group showed significant decrease of serum CPK as compared to PD+Vin group. Serum CPK was significantly lower in PD+Pir\&Vin group versus
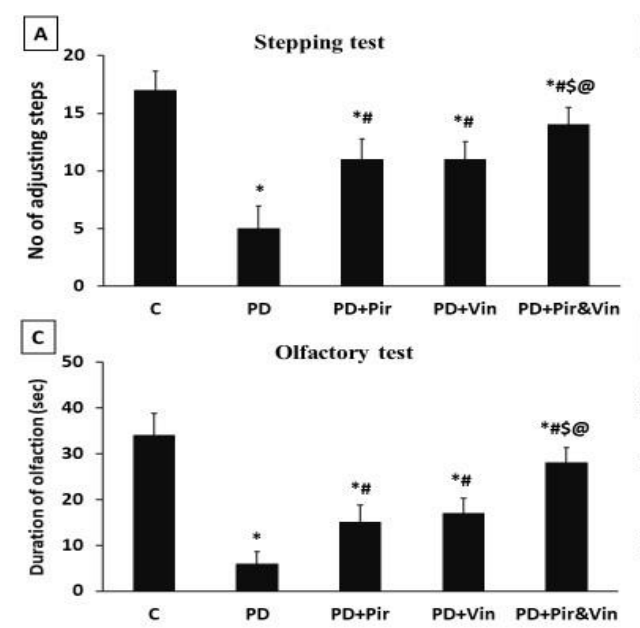

Figure (1): Neurological and behavioral test results in control (C), Parkinson's disease (PD), piracetam-treated Parkinson's disease (PD+Pir), vincamine-treated Parkinson's disease (PD+Vin) and piracetam- \& vincamine-treated Parkinson's disease (PD+Pir\&Vin) groups. Neurological and behavioral assessments were done as described in Materials and Methods. Results are expressed as mean \pm standard deviation $(\mathrm{SD})$. Number of rats in each group was eight $(\mathrm{n}=8)$. Data were statistically analyzed by oneway ANOVA, and the level of significance was set on $\mathrm{P} \leq 0.05$. *: significant versus $\mathrm{C}$ group, \#: significant versus $\mathrm{PD}$ group, $\$$ : significant versus PD+Pir group, @: significant versus PD+Vin group.

Table (1): Serum fasting glucose, calcium, creatine phosphokinase (CPK) and glial cell-derived neurotrophic factor (GDNF) levels in control (C), Parkinson's disease (PD), piracetam-treated Parkinson's disease (PD+Pir), vincaminetreated Parkinson's disease (PD+Vin) and piracetam- \& vincamine-treated Parkinson's disease (PD+Pir\&Vin) groups:

\begin{tabular}{|c|c|c|c|c|c|}
\hline Group & $\bar{C}$ & PD & PD+Pir & PD+Vin & PD+Pir\&Vin \\
\hline Serum glucose (mg/dl) & $87.82 \pm 4.68$ & $191.42 \pm 4.66^{*}$ & $105.12 \pm 4.84^{* \#}$ & $107.85 \pm 4.82^{\text {*\# }}$ & $102.97 \pm 5.07^{* \# \#}$ \\
\hline Serum calcium (mg/dl) & $9.90 \pm 0.45$ & $5.83 \pm 0.53^{*}$ & $8.77 \pm 0.58^{* \#}$ & $8.60 \pm 0.51^{* \#}$ & $8.93 \pm 0.59^{* \# @ ~}$ \\
\hline Serum CPK (U/ml) & $124.27 \pm 7.5$ & $411.5 \pm 11.2^{*}$ & $168.08 \pm 3.2^{* \#}$ & $193.57 \pm 6.4^{* \# \$}$ & $142.17 \pm 4.2^{* \# \$ @ ~}$ \\
\hline Serum GDNF (pg/ml) & $129.87 \pm 6.57$ & $50.83 \pm 8.19^{*}$ & $97.28 \pm 6.19^{* \#}$ & $94.53 \pm 5.91^{* \#}$ & $112.73 \pm 5.74^{* \# \$ @ ~}$ \\
\hline
\end{tabular}

Biochemical parameters were measured as described in Materials and Methods. Results are expressed as mean \pm standard deviation (SD). Number of rats in each group was eight $(\mathrm{n}=8)$. Data were statistically analyzed by one-way ANOVA, and the level of significance was set on $\mathrm{P} \leq 0.05$. *: significant versus $\mathrm{C}$ group, \#: significant versus PD group, $\$$ : significant versus PD+Pir group, @: significant versus PD+Vin group. 


\subsubsection{Striatal biochemical assays.}

Table (2) demonstrates the various biochemical parameters measured in the supernatants of the striatal tissue homogenates of all the studied groups.

Regarding striatal homogenate dopamine level, there was a significant difference among the studied groups. Dopamine level was significantly lower in PD group than in $\mathrm{C}$ group. In PD+Pir, $\mathrm{PD}+\mathrm{Vin}$ and $\mathrm{PD}+\mathrm{Pir} \& \mathrm{Vin}$ groups, dopamine levels were significantly higher than in PD group, but they were still significantly lower than in $\mathrm{C}$ group. There was insignificant difference between dopamine levels in $\mathrm{PD}+\mathrm{Pir}$ and $\mathrm{PD}+\mathrm{Vin}$ groups, however, dopamine level in PD+Pir\&Vin group was significantly higher than in any one of them.

Regarding oxidative stress markers, there were significant differences in striatal MDA and nitrite/nitrate levels among the studied groups. Striatal MDA and nitrite/nitrate levels in PD group were significantly higher than those in $\mathrm{C}$ group. Both MDA and nitrite/nitrate levels in PD+Pir, $\mathrm{PD}+\mathrm{Vin}$ and $\mathrm{PD}+\mathrm{Pir} \& V i n$ groups were significantly reduced, when compared with those in PD group, but they were still significantly more than those in $\mathrm{C}$ group. The MDA level was significantly less in $\mathrm{PD}+\mathrm{Vin}$ group than in $\mathrm{PD}+\mathrm{Pir}$ group, while a nonsignificant variation in nitrite/nitrate level was observed between the two groups. Both striatal MDA and nitrite/nitrate levels in PD+Pir\&Vin group were significantly decreased, when compared with corresponding values in $\mathrm{PD}+\mathrm{Pir}$ and $\mathrm{PD}+\mathrm{Vin}$ groups.

Regarding the antioxidants in striatal tissue homogenates, there were significant differences in the GSH level, and the GPx and SOD activities among the studied groups. The GSH level, and the
GPx and SOD activities in PD group were significantly less than those in $\mathrm{C}$ group. All the above-mentioned antioxidants were significantly elevated in PD+Pir, PD+Vin and PD+Pir\&Vin groups, when compared with the corresponding values in PD group, but they were still significantly less than those in $\mathrm{C}$ group. $\mathrm{PD}+\mathrm{Vin}$ group showed a significantly higher level of GSH, and activities of GPx and SOD than the corresponding values in $\mathrm{PD}+\mathrm{Pir}$ group. PD+Pir\&Vin group showed significant increase in GSH, GPx and SOD, when compared with those in PD+Pir group, but were insignificantly changed from those in $\mathrm{PD}+\mathrm{Vin}$ group.

The striatal levels of the proinflammatory markers, TNF- $\alpha$ and IL-1ß, were significantly higher in PD group than in $\mathrm{C}$ group. In the treated groups (PD+Pir, $\mathrm{PD}+\mathrm{Vin}$ and $\mathrm{PD}+\mathrm{Pir} \& \mathrm{Vin}$ groups), both TNF- $\alpha$ and IL-1ß levels were significantly reduced, when compared with PD group, but they were still significantly more than those in C group. The TNF- $\alpha$ and IL-1ß levels were significantly less in PD+Pir group than in $\mathrm{PD}+\mathrm{Vin}$ group, and both levels were significantly less in PD+Pir\&Vin group than in PD+Pir and PD+Vin groups.

\subsection{Correlation between Striatal dopamine level and other measured variables.}

Table (3) shows the Pearson correlations of the dopamine level in the striatal tissue homogenate with the other studied variables.

Significant positive correlations were observed between the striatal dopamine level and the number of adjusting steps in stepping test, percentage of spontaneous alternations in Y-maze test, duration of olfaction in the olfactory test and latency time in swimming test for the control and 
all the Parkinson's disease studied groups of rats in one analysis; a significant negative correlation of dopamine level with immobility time in swimming test was shown.

Also, there were significant positive correlations between the striatal dopamine level and the serum calcium, serum GDNF and striatal antioxidants (GSH level, GPx and SOD activities) of all studied groups in one analysis.

On the other hand, striatal dopamine level showed significant negative correlations with the serum glucose and CPK levels, oxidant markers, MDA and nitrite/nitrate level, and the striatal proinflammatory markers, TNF- $\alpha$ and IL- $1 \beta$, of all studied groups in one analysis.

Table (2): Dopamine, malondialdehyde (MDA), nitrite/nitrate, reduced glutathione (GSH), tumor necrosis factor-alpha (TNF- $\alpha$ ) and interleukin-1 beta (IL-1ß) levels, and the glutathione peroxidase (GPx) and superoxide dismutase (SOD) enzymatic activities in striatal homogenates of control (C), Parkinson's disease (PD), piracetam-treated Parkinson's disease (PD+Pir), vincamine-treated Parkinson's disease (PD+Vin) and piracetam- \& vincamine-treated Parkinson's disease (PD+Pir\&Vin) groups:

\begin{tabular}{|c|c|c|c|c|c|}
\hline Group & $\mathrm{C}$ & PD & PD+Pir & PD+Vin & PD+Pir\&Vin \\
\hline $\begin{array}{l}\text { Dopamine } \\
\text { (ng/g tissue) }\end{array}$ & $0.40 \pm 0.07$ & $0.06 \pm 0.05^{*}$ & $0.22 \pm 0.05^{* \#}$ & $0.21 \pm 0.03^{* \#}$ & $0.33 \pm 0.05^{* \# \$}$ \\
\hline $\begin{array}{l}\text { MDA } \\
\text { (nmol/g tissue) }\end{array}$ & $2.29 \pm 0.28$ & $12.22 \pm 0.64^{*}$ & $5.68 \pm 0.46^{* \#}$ & $3.54 \pm 0.47^{\text {*\#\$ }}$ & $2.99 \pm 0.51^{* \# \$ @}$ \\
\hline $\begin{array}{l}\text { Nitrite/nitrate } \\
\text { ( } \mu \mathrm{mol} / \mathrm{g} \text { tissue })\end{array}$ & $11.67 \pm 5.5$ & $31.33 \pm 6.06^{*}$ & $23.17 \pm 4.17^{\text {*\# }}$ & $21.83 \pm 4.07^{\text {*\# }}$ & $17.83 \pm 4.02^{* \# \$ @}$ \\
\hline $\begin{array}{l}\text { GSH } \\
\text { (mg/g tissue) }\end{array}$ & $66.77 \pm 5.74$ & $19.55 \pm 2.46^{*}$ & $31.6 \pm 5.18^{* \#}$ & $55.72 \pm 6.17^{* \# \$}$ & $56.90 \pm 6.48^{* \# \$}$ \\
\hline $\begin{array}{l}\text { SOD } \\
\text { (U/g tissue) }\end{array}$ & $64.85 \pm 4.57$ & $23.95 \pm 3.7^{*}$ & $34.42 \pm 5.76^{\text {*\# }}$ & $54.59 \pm 6.62^{* \# \$}$ & $54.37 \pm 5.49^{* \# \$}$ \\
\hline $\begin{array}{l}\text { TNF- } \alpha \\
\text { (ng/g tissue) }\end{array}$ & $54.03 \pm 2.15$ & $102.99 \pm 11.1^{*}$ & $66.79 \pm 3.87^{\text {*\# }}$ & $80.56 \pm 3.52^{\text {*\#\$ }}$ & $60.16 \pm 3.67^{* \# \$ @}$ \\
\hline $\begin{array}{l}\text { IL-1ß } \\
\text { (pg/g tissue) }\end{array}$ & $23.92 \pm 4.06$ & $124.18 \pm 30^{*}$ & $40.95 \pm 6.74^{* \#}$ & $72.08 \pm 5.91^{* \# \$}$ & $32.28 \pm 5.97^{\text {*\#\$@ }}$ \\
\hline
\end{tabular}

Biochemical parameters were measured as described in Materials and Methods. Results are expressed as mean \pm standard deviation (SD). Number of rats in each group was eight $(n=8)$. Data were statistically analyzed by one-way ANOVA, and the level of significance was set on $\mathrm{P} \leq 0.05$. *: significant versus $\mathrm{C}$ group, \#: significant versus $\mathrm{PD}$ group, \$: significant versus PD+Pir group, @: significant versus PD+Vin group.

Table (3): Pearson correlation coefficient (r) between striatal dopamine level and the neurological and behavioral assessments for all rats $(n=40)$ :

\begin{tabular}{cccccc}
\hline & $\begin{array}{c}\text { number of } \\
\text { adjusting steps }\end{array}$ & $\begin{array}{c}\text { Y-maze score }(\% \text { of } \\
\text { alternation) }\end{array}$ & $\begin{array}{c}\text { olfactory } \\
\text { test }\end{array}$ & \multicolumn{2}{c}{ swimming test } \\
\cline { 4 - 6 } Dopamine (r) & 0.880 & 0.821 & 0.893 & 0.897 & -0.909 \\
tatency & $\begin{array}{c}\text { immobility } \\
\text { time }\end{array}$ & \\
\hline
\end{tabular}

\footnotetext{
Pearson correlation was significant $(\mathrm{P}<0.001)$ for all parameters.
} 
Table (4): Pearson correlation coefficient (r) between striatal dopamine level and the measured biochemical variables for all rats $(n=40)$ :

\begin{tabular}{|c|c|c|c|c|c|c|c|c|c|c|c|}
\hline & \multicolumn{4}{|c|}{ serum } & \multicolumn{7}{|c|}{ striatal tissue homogenate } \\
\hline & glucose & calcium & CPK & GDNF & MDA & $\begin{array}{c}\text { nitrite/ } \\
\text { nitrate }\end{array}$ & GSH & GPx & SOD & TNF- $\alpha$ & IL-1B \\
\hline $\begin{array}{c}\text { Dopamine } \\
\text { (r) }\end{array}$ & -0.774 & 0.843 & -0.768 & 0.842 & -0.760 & -0.753 & 0.746 & 0.777 & 0.816 & -0.781 & -0.780 \\
\hline
\end{tabular}

CPK: creatine phosphokinase, GDNF: glial derived neurotrophic factor, MDA: malondialdehyde, GSH: reduced glutathione, GPx: glutathione peroxidase, SOD: superoxide dismutase, TNF- $\alpha$ : tumor necrosis factor-alpha, IL-1ß: interleukin-1 beta. Pearson correlation was significant $(\mathrm{P}<0.001)$ for all parameters.

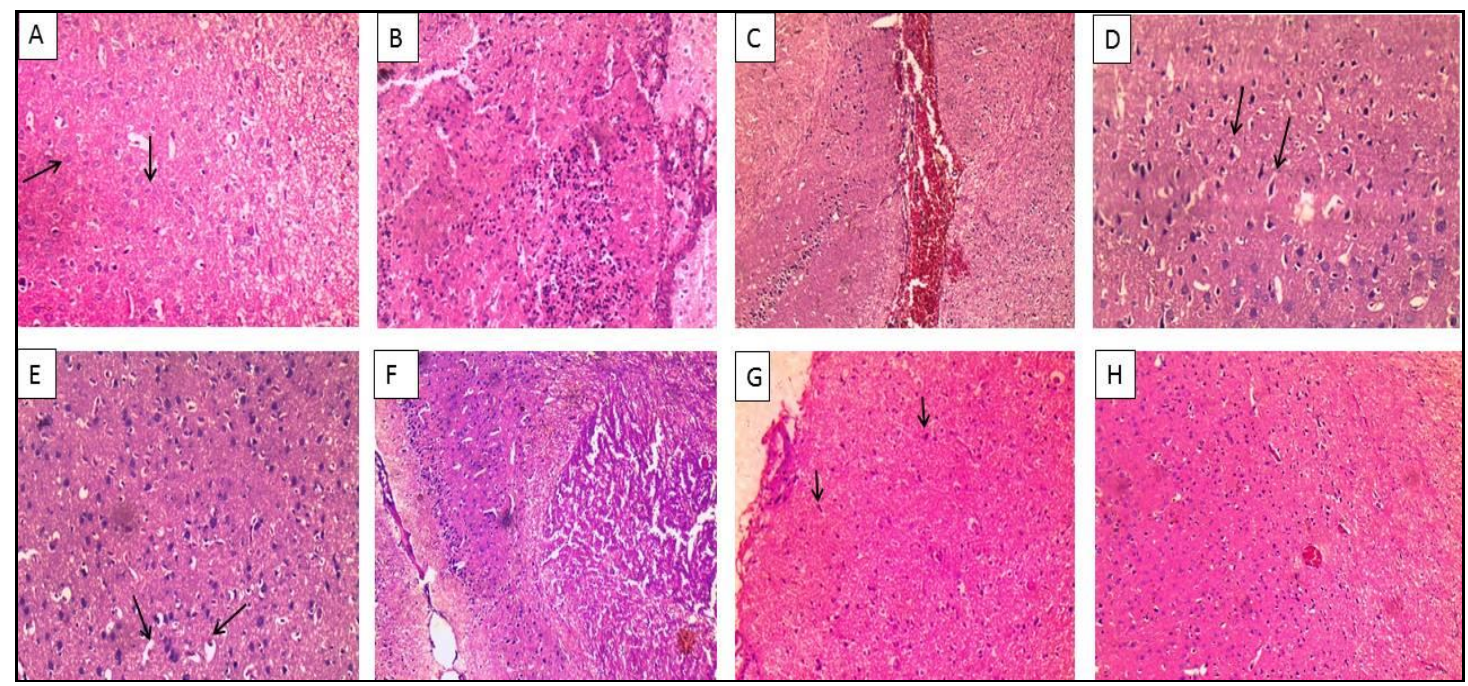

Figure (2): Photo micrographs of Hematoxylin and eosin (H\&E)-stained sections of substantia nigra pars compacta (SNpc) (×200) from: (A) control group showing the normal histological picture of SNpc neurons with vesicular nuclei, prominent nucleoli and scant rim of basophilic cytoplasm. (B) Parkinson's disease group showing extensive lymphoplasmacytic inflammatory infiltration. (C) Parkinson's disease group showing extensive hemorrhage and congested blood vessels. (D) Parkinson's disease group showing neuronal degeneration in which neurons appeared with shrunken nuclei, inconspicuous nucleoli and surrounded with clear halo. (E) Parkinson's disease group showing numerous Lewis bodies (cytoplasmic eosinophilic inclusions). (F) piracetam-treated Parkinson's disease group showing gliosis and regression of inflammatory infiltrate. (G) vincamine-treated Parkinson's disease group showing mild vascular congestion and numerous regenerating neurons. (H) piracetam- and vincamine-treated Parkinson's disease group showing marked regression of both inflammation and congestion, with numerous regenerating neurons.

\section{Discussion}

Chronic haloperidol administration to rats for four weeks induced PD-like features [20] that was confirmed in the current study by the presence of bradykinesia, cognitive impairment, depressive-like behavior and hyposmia. The impaired neurological and behavioral tests in haloperidol-administered rats could be attributed to our observation of significant decrease in the striatal dopamine level, compared to normal animals; findings which are consistent with Amato et al. [21]. Loss of dopaminergic neurons in haloperidol-administered rats was demonstrated in this investigation by the histopathological changes in substantia nigra, which is consistent with the observation of Abdelwahab et al. [22]. The impaired neurological and behavioral tests in PD rats were ameliorated in haloperidol-administered animals co-treated with piracetam or vincamine, and the combination of both agents exerted better improvement than treatment with each one alone.

Bradykinesia in PD rats was evidenced by decreased number of adjusting steps in stepping test, compared to normal animals; this could be attributed to a decrease of dopamine level in striatal tissue [23], as confirmed by presence of positive 
correlation between striatal dopamine and stepping test. We observed a significant improvement in striatal dopamine in animals receiving piracetam or vincamine with haloperidol injection. In agreement, Zaitone et al. [24] have reported that vinpocetine, a synthetic derivative of vincamine, enhances the motor activity in a PD-rat model by restoring dopamine level in corpus striatum.

As dopamine is essential for working memory [25], we used Y-maze for its assessment. Our data showed that decreased dopamine level correlates the impairment of working memory with a reduction in percentage of spontaneous alternation; a finding that is in line with Handra et al. [26]. Treatment with either piracetam or vincamine improved the haloperidol-induced memory deficit. In agreement with the present results, Abdel-Salam and Nada [27] observed that piracetam alleviates the antipsychotic-induced impairment of learning and memory. Vincamine has been shown to improve speed of memory learning and recall in both cognitively healthy subjects and compromised subjects [28].

Rats with PD showed depressive-like behavior, as evidenced by increased immobility time of swimming test, when compared with normal animals. In fact, depression associates dopaminergic system dysfunction [29] that was indicated in the present study by the presence of negative correlation between striatal dopamine and immobility time of swimming test. An antidepressive effect was exerted by piracetam or vincamine, which is probably due to their neuroprotective effect, as evidenced by improvement in GDNF and increasing dopamine level in the striatal tissue. Piracetam is also a positive modulator of glutamate receptors and it increases excitatory neurotransmission [30]. Vincamine is claimed to improve memory impairment accompanying neurodegenerative diseases by its antioxidant effect, increasing cerebral blood flow and glucose and oxygen utilization by the neurons [31].

We noted a positive correlation between dopamine level and duration of olfaction, reflecting role of dopamine in the olfactory process. Olfactory impairment in PD may be due to defective adult neurogenesis that can be modulated by dopamine [32]. Haloperidol-induced hyposmia was alleviated by treatment with piracetam, vincamine or both. The neuroprotective effect of piracetam could be explained by its ability to enhance membrane fluidity, especially that of mitochondria [33]. Restoring membrane fluidity promotes neurotransmission and enhances neuroplasticity [9]. We measured serum glucose level to study the effect of haloperidol and the used treated drugs on it, as observed from previous studies hyperglycemic effect of haloperidol [34], that could be corrected with piracetam and vincamine treatment $[35,36]$. The findings of the current study revealed a significant increase of serum glucose in haloperidol-administered rats, as compared to normal ones. Chen et al. [34] had attributed the hyperglycemic effect of haloperidol due to induction of metabolic disturbances, insulin resistance, pancreatic cell dysfunction, dyslipidemia, and weight gain. Treatment with piracetam and vincamine improved the hyperglycemic state. In agreement with our results, Can et al. [35] reported improvements of hyperglycemia and cognitive dysfunction in diabetic patients upon treatment with piracetam, and explained their observation by an increase of 
glucose uptake and oxidation in the neurons. Also, vincamine displays hypoglycemic effect by increasing cerebral blood flow and glucose utilization [36].

Disturbance of calcium homeostasis is supposed to be another key factor in the pathogenesis of PD [37]. A reduction of serum calcium level was observed in PD group, compared to normal rats. Co-administration of piracetam, vincamine or both with haloperidol was found to increase serum calcium. In fact, chronic administration of haloperidol encourages calcium influx via L-type calcium channels [38]. Both piracetam and vincamine act as calcium-channel blockers, so they reduce cellular calcium influx and preserve calcium in plasma [39].

CPK is an intracellular enzyme present in our body in heart, brain, and skeletal muscles, and other sites. It leaks into blood when muscle damage occurs, therefore high level of CPK denoting some sort of stress or injury affecting muscle leading to proteolysis and increasing membrane permeability [40], so, in this work we measured serum CPK level which found to be significantly increased after haloperidol administration, reflecting muscle damage [40], a finding which was in consistency with a previous study [12]. Serum CPK was reduced by co-administration of piracetam or vincamine with haloperidol, as both agents improve motor function and reduce haloperidol effect on skeletal muscles.

We measured serum GDNF as, it if found that it protects dopaminergic neurons in animal models of $\mathrm{PD}$, and rescues motor neurons in vivo, raising hopes that it could be used as a therapeutic agent to treat several neurodegenerative diseases [41]. There was a decrease in serum GDNF in non-treated PD rats, as compared to control animals. In support with our finding, Hisaoka et al. [42] proved that haloperidol decreases GDNF release from rat C6 glioma cells. In contrast, Amin et al. [12] reported an increase in GDNF level in haloperidoladministered rats. They explained their contradictory result by a possible up-regulation of GDNF after nigral neuronal injury. Coadministration of piracetam or vincamine with haloperidol resulted in an increase in serum GDNF. Oxidative stress is implicated in the pathogenesis of many neurodegenerative disorders, including PD [43]. Administration of haloperidol to rats resulted in an elevation of MDA, one of the major lipid peroxidation products, and total nitrite/nitrate levels, with concomitant reductions in the antioxidant GSH level and the GPx and SOD enzymatic activities in their striatal tissue, as compared to normal rats. These results agree with Zaidi et al. [44], who reported elevation of the oxidative stress and neuro-inflammatory markers in haloperidol-induced PD. Our data demonstrate a positive correlation of dopamine with antioxidants and a negative correlation with MDA and total nitrite/nitrate, denoting the role of oxidative stress in PD development and progression and the protective role of the antioxidant agents.

Haloperidol-induced oxidative stress was mitigated by treatment with piracetam or vincamine, as indicated by lower MDA and nitrite/nitrate and higher antioxidants in treated PD groups versus non-treated one, signifying an antioxidant function of both piracetam and vincamine that is in accordance with observations in other studies [28, 36]. In fact, both piracetam and vincamine preserve and protect dopaminergic neurons in a PD rat model by several mechanisms including antioxidant 
and anti-inflammatory properties [24]. Piracetam enhances mitochondrial membrane fluidity by enhancing the function of the respiratory chain complexes [33]. Vincamine has an antioxidant scavenging capacity; it inactivates hydroxyl free radicals, in addition to its capability to prevent iron accumulation in rat brain [31].

Neuro-inflammation plays an important role in PD pathogenesis [45]. In consistency with previous studies [44, 46], haloperidol injection resulted in increases of the proinflammatory cytokines, TNF- $\alpha$ and IL1- $\beta$, in rat brain homogenate. Our data showed that co-administration of piracetam or vincamine to haloperidol-administered rats results in reduction of striatal levels of these proinflammatory cytokines, when compared with PD animals which did not receive any treatment, denoting an anti-inflammatory effect of both agents. Previous studies supported the antiinflammatory effect of piracetam [24] and vincamine [47], which gives a new aspect about the role of nootropic drugs in the treatment of PD. However, Zaitone et al. [24] denies any significant effect of vinpocetine, the derivative of vincamine, on striatal TNF- $\alpha$ level. Interestingly, there was a significant negative correlation between the measured proinflammatory markers (TNF- $\alpha \&$ IL1B) and dopamine level in our work, indicating that they play a role in sustaining dopaminergic degeneration in $\mathrm{PD}$, and denoting the role of neuroinflammation in PD development and progression, and the protective role of the anti-inflammatory agents.

Neurodegeneration observed in histopathological finding in PD group may be due to inflammatory and oxidative stress state mediated by haloperidol administration that was mitigated by piracetam and vincamine. Both agents improved histopathological changes in substantia nigra of PD rats, that was also seen by Zaitone et al. [24], possibly by increasing blood supply to the brain, delivering nutrient and energy required for neuronal function, improving neurotransmitter release, and having antioxidant and anti-inflammatory function [48].

The hallmark of our findings is that improvement of oxidative stress markers was more significant when treating PD rats with vincamine than piracetam, while reduction of pro-inflammatory cytokines was better with piracetam than vincamine. Striatal MDA level was lower, while the antioxidants GSH, GPx and SOD were higher in vincamine-treated PD rats than in piracetamtreated ones. The reduction of striatal TNF- $\alpha$ and IL1- $\beta$ levels was more significant when treating PD rats with piracetam than vincamine. These results suggest that the neuroprotective effect of piracetam in haloperidol-induced PD arises more from its anti-inflammatory properties, whereas vincamine is more likely to achieve its protective effect via its antioxidant action.

The major finding of the present study is that the combined administration of both piracetam and vincamine to the haloperidol-induced $\mathrm{PD}$ rats ameliorated the associated deleterious effects better than administration of each one alone. Combined piracetam and vincamine administration to PD rats improved neurological and behavioral performance and serum calcium, CPK and GDNF levels better than in isolated piracetam or vincamine administration. Also, Striatal dopamine, MDA, nitrite/nitrate, TNF- $\alpha$ and IL1- $\beta$ levels showed better improvement in combined treatment than in case of treatment with either piracetam or vincamine alone. The better amelioration of the 
neurological and biochemical errors by the combined administration of piracetam and vincamine could be explained by an additive effect, as the two agents belong to distinct chemical classes and have different mechanisms of action. However, potential adverse effects of using the two agents in combination may exist, and should be investigated to ensure that their combination has a beneficial effect in protection of the detrimental effects of PD.

Limitations to this study, doses of used nootropic drugs, that we recommend further studies to assess the effect of different doses of these drugs. Also, more techniques like immunohistochemistry and PCR may be more helpful in such work.

In conclusion, both piracetam and vincamine exhibit neuroprotective effect in haloperidolinduced PD rat model and modulate striatal dopamine release, and thus could be considered as possible candidates for management of PD. The combination of both nootropic agents affords a better protection than the use of each one alone that could be explained by an additive effect based on their different mechanisms of action. Piracetam reaches its effect mainly by its anti-inflammatory action, while vincamine has mainly antioxidant properties. It is recommended to study these agents thoroughly in clinical trials for use in $\mathrm{PD}$, and to investigate possible adverse effects of their combination, if any.

\section{Conflict of interest:}

The authors declare that there is no conflict of interest associated with this work.
We would like to thank Dr. Alyaa Abo-Elmagd, Pathology Department, Menoufia Faculty of Medicine, Egypt, for performing and interpretation of the histological study.

\section{References}

1. Hirsch L, Jette N, Frolkis A, Steeves T, Pringsheim T. The incidence of Parkinson's disease: a systematic review and metaanalysis. Neuroepidemiology 46(4): 292-300, 2016.

2. Kikuchi T, Morizane A, Doi D, Magotani H, Onoe H, Hayashi T, Mizuma H, Takara S, Takahashi R, Inoue H, Morita S. Human iPS cell-derived dopaminergic neurons function in a primate Parkinson's disease model. Nature 548(7669): 592-596, 2017.

3. Jellinger KA. Neuropathology of nonmotor symptoms of Parkinson's disease. Academic Press Int Rev Neurobiol 133: 13-62, 2017.

4. Schapira AH, Chaudhuri KR, Jenner P. Non-motor features of Parkinson disease. Nat Rev Neurosci 18(7): 435, 2017.

5. Hatcher JM, Pennell KD, Miller GW. Parkinson's disease and pesticides. a toxicological perspective. Trends Pharmacol Sci 1;29(6): 322-329, 2008.

6. Trinh J, Farrer M. Advances in the genetics of Parkinson disease. Nat Rev Neurol 8: 445454, 2013.

7. Ward KM, Citrome L. Antipsychotic-related movement disorders: Drug-induced parkinsonism vs. tardive dyskinesia-Key differences in pathophysiology and clinical management. Neurol Ther 7(2): 233-248, 2018.

\section{Acknowledgment}


8. López-Sendón J, Mena MA, G de Yébenes

J. Drug-induced parkinsonism. Expert Opin Drug Saf 12(4): 487-496, 2013.

9. Verma DK, Gupta S, Biswas J, Joshi N, Singh A, Gupta P, Tiwari S, Raju KS, Chaturvedi S, Wahajuddin M, Singh S. New therapeutic activity of metabolic enhancer piracetam in treatment of neurodegenerative disease: Participation of caspase independent death factors, oxidative stress, inflammatory responses and apoptosis. Biochim Biophys Acta Mol Basis Dis 1864(6): 2078-2096, 2018.

10. Fayed AH. Brain trace element concentration of rats treated with the plant alkaloid, vincamine. Biol Trace Elem Res 136: 314$319,2010$.

11. Kashihara K, Sato M, Fujiwara Y, Harada T, Ogawa T, Otsuki S. Effects of intermittent and continuous haloperidol administration on the dopaminergic system in the rat brain. Biol Psychiatry 21(7): 650-656, 1986.

12. Amin SN, Al Okda AM, Rashed LA. Neuroprotective Effects of Piracetam Versus Peroxisome Proliferator-Activated ReceptorGamma Agonist Pioglitazone in Drug-Induced Parkinsonism. Group.;5:1ml. International Annals of Medicine 1(4): 2017.

13. Kanig K, Hoffmann KH. 32P-incorporation in adenosine phosphates of the rat brain after oral application of vincamine for 2 weeks (author's transl). Arzneimittelforschung 29(1): 33-34, 1979.

14. Paillé V, Henry V, Lescaudron L, Brachet P, Damier P. Rat model of Parkinson's disease with bilateral motor abnormalities, reversible with levodopa, and dyskinesias. Mov Disord 22(4):533-539, 2007.

15. Amin SN, El-Aidi AA, Ali MM, Attia YM, Rashed LA. Modification of hippocampal markers of synaptic plasticity by memantine in animal models of acute and repeated restraint stress: implications for memory and behavior. Neuromolecular Med 17(2): 121-136, 2015.

16. Pesarico AP, Sampaio TB, Stangherlin EC, Mantovani AC, Zeni G, Nogueira CW. The antidepressant-like effect of 7-fluoro-1, 3diphenylisoquinoline-1-amine in the mouse forced swimming test is mediated by serotonergic and dopaminergic systems. Prog Neuropsychopharmacol Biol Psychiatry 54: 179-186, 2014.

17. Tillerson JL, Caudle WM, Parent JM, Gong C, Schallert T, Miller GW. Olfactory discrimination deficits in mice lacking the dopamine transporter or the D2 dopamine receptor. Behav Brain Res 172(1):97-105, 2006.

18. Bancroft JD, Gamble M, editors. Theory and practice of histological techniques. Elsevier health sciences, 2008.

19. Altman DG Sim. Statistics in medical journals: developments in the 1980s. Statistics in Medicine 10(12):1897-1913, 1991.

20. Pingale T, Gupta GL. Classic and evolving animal models in Parkinson's disease. Pharmacol Biochem Behav 199: 173060, 2020.

21. Amato D, Natesan S, Yavich L, Kapur S, Müller CP. Dynamic regulation of dopamine and serotonin responses to salient stimuli during chronic haloperidol treatment. Int $J$ Neuropsychopharmacol 14(10):1327-1339, 2011. 
22. Abdelwahab SA, Elsebaay SA, Ibrahim MF, Abdel-Hafez SM. Histological changes in the substantia nigra in Parkinsonism model in male albino rat. MJMR 30(4): 193-197, 2019.

23. Khan MM, Ahmad A, Ishrat T, Khan MB, Hoda MN, Khuwaja G, Raza SS, Khan A, Javed H, Vaibhav K, Islam F. Resveratrol attenuates 6-hydroxydopamine-induced oxidative damage and dopamine depletion in rat model of Parkinson's disease. Brain research 1328: 139-151, 2010.

24. Zaitone SA, Abo-Elmatty DM, Elshazly SM. Piracetam and vinpocetine ameliorate rotenone-induced Parkinsonism in rats. Indian J Pharmacol 44(6): 774-779, 2012.

25. Xu H. Chronic Administration of Haloperidol and Working Memory: Roles of Caudate Putamen Dopamine Receptors. Curr Neurobiol 8(2): 27-33, 2017.

26. Handra C, Coman OA, Coman LA, Enache T, Stoleru S, Sorescu AM, Ghită I, Fulga I. The connection between different neurotransmitters involved in cognitive processes. Farmacia 67(2): 193-201, 2019.

27. Abdel-Salam OM, Nada SA. Effect of piracetam, vinpocetine and ginkgo biloba on antipsychotic-induced impairment of learning and memory. Cesk Slov Neurol 74: 29-35, 2011.

28. Abdel Salam OM, Oraby FH, Hassan NS. Vinpocetine ameliorates acute hepatic damage caused by administration of carbon tetrachloride in rats. Acta Biol Hung 4: 411419, 2007.

29. León LA, Cardenas FP. Contribution of the dopaminergic system to the effect of chronic fluoxetine in the rat forced swim test. Psychol Neurosci 1(1): 81-86, 2008.

30. Gokhale VS, Bhide SS, Jalgaonkar SV, Marathe PA, Mane Y, Khan FM, Rege NN. Evaluation of effect of Piracetam in experimental models of Depression. Int $J$ Pharm Sci Res 4(7): 2667-2672, 2013.

31. Han J, Qu Q, Qiao J, Zhang J. Vincamine alleviates amyloid- $\beta$ 25-35 peptides-induced cytotoxicity in PC12 cells. Phog Mag 13: 123-128, 2017.

32. Höglinger GU, Rizk P, Muriel MP, Duyckaerts C, Oertel WH, Caille I, Hirsch EC. Dopamine depletion impairs precursor cell proliferation in Parkinson disease. Nat Neurosci 7(7): 726-735, 2004.

33. Leuner K, Kurz C, Guidetti G, Orgogozo JM, Müller WE. Improved mitochondrial function in brain aging and Alzheimer diseasethe new mechanism of action of the old metabolic enhancer piracetam. Front neurosci 4: 44, 2010.

34. Chen J, Huang XF, Shao R, Chen C, Deng C. Molecular mechanisms of antipsychotic drug-induced diabetes. Front Neurosci 11: 643, 2017.

35. Can OD, Üçel UI, Özkay UD, Ulupinar E. The Effect of Agomelatine Treatment on Diabetes-Induced Cognitive Impairments in Rats: Concomitant Alterations in the Hippocampal Neuron Numbers. Int J Mol Sci 19(8): 2461, 2018.

36. Nandini HS, Naik PR. Antidiabetic, antihyperlipidemic and antioxidant effect of Vincamine, in streptozotocin-induced diabetic rats. Eur J Pharmacol 843: 233-239, 2019. 
37. Surmeie DJ. Calcium, ageing and neuronal vulnerability in Parkinson's disease. Lancet Neurol 6:933-938, 2007.

38. Kim HS, Yumkham S, Choi JH, Kim EK, Kim YS, Ryu SH, Suh PG. Haloperidol induces calcium ion influx via L-type calcium channels in hippocampal HN33 cells and renders the neurons more susceptible to oxidative stress. Mol Cells 22(1): 51-57, 2006.

39. Onose G, Daia-Chendreanu C, Haras M, Ciurea AV, Anghelescu A. Traumatic brain injury: Current endeavours and trends for neuroprotection and related recovery. Romanian Neurosurgery: 11-30, 2011.

40. Bektas H, Deniz O, Temel S, Keklikoglu HD, Akyol S. Rhabdomyolysis related to dyskinesia in Parkinson's disease. $J$ Mov Disord 7: 25-27, 2014.

41. Padmakumar S, Taha M, Kadakia E, Bleier B, Amiji M. Delivery of neurotrophic factors in the treatment of age-related chronic neurodegenerative diseases. Expert Opinion on Drug Delivery 17(3): 323-340, 2020.

42. Hisaoka K, Nishida A, Koda T, Miyata M, Zensho H, Morinobu S, Ohta M, Yamawaki S. Antidepressant drug treatments induce glial cell line-derived neurotrophic factor (GDNF) synthesis and release in rat C6 glioblastoma cells. J Neurochem 79(1): 25-34, 2001.

43. Nigam D. Free radicals and oxidative stress in neurodegenerative disorders. In Free radicals in human health and disease. Springer, New Delhi: 143-158, 2015

44. Zaidi A, Khan M, Shahreyar Z, Ahmed H. Lauric Acid: Its Role in Behavioral Modulation, Neuro-inflammatory and Oxidative Stress Markers in Haloperidol
Induced Parkinson's Disease. Pak J Pharm Sci 33(2 supplementary): 755-763, 2020.

45. Hirsch EC, Vyas S, Hunot S. Neuroinflammation in Parkinson's disease. Parkinsonism Relat Disord 18: S210-212, 2012.

46. Abdel-Salam OM, Sleem AA, Youness ER, Mohammed NA, Omara EA. Bone MarrowDerived Protect Against Haloperidol-Induced Brain and Liver Damage in Mice. Biomed Pharmacol J 11(1):11-22 2018.

47. Wu L, Ye M, Zhang J. Vincamine prevents lipopolysaccharide induced inflammation and oxidative stress via thioredoxin reductase activation in human corneal epithelial cells. Am J Transl Res 10(7):2195-2204, 2018.

48. Suliman NA, Taib CN, Moklas MA, Adenan MI, Baharuldin MT, Basir R. Establishing Natural Nootropics: Recent Molecular Enhancement Influenced by Natural Nootropic. Evid Based Complement Alternat Med: Article ID 4391375, 12 pages, 2016. 